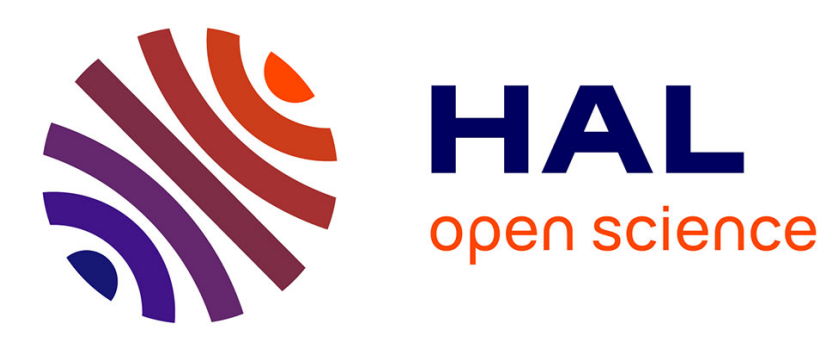

\title{
A Shock Filter for Image Deblurring and Enhancement with Oriented Hourglass Tensor
}

\author{
Baptiste Magnier, Behrang Moradi, Cedric Walbrecq
}

\section{To cite this version:}

Baptiste Magnier, Behrang Moradi, Cedric Walbrecq. A Shock Filter for Image Deblurring and Enhancement with Oriented Hourglass Tensor. ISPA 2019 - 11th International Symposium on Image and Signal Processing and Analysis, Sep 2019, Dubrovnik, Croatia. pp.111-116, 10.1109/ISPA.2019.8868552 . hal-02436586

\section{HAL Id: hal-02436586 https://hal.mines-ales.fr/hal-02436586}

Submitted on 3 Jun 2021

HAL is a multi-disciplinary open access archive for the deposit and dissemination of scientific research documents, whether they are published or not. The documents may come from teaching and research institutions in France or abroad, or from public or private research centers.
L'archive ouverte pluridisciplinaire HAL, est destinée au dépôt et à la diffusion de documents scientifiques de niveau recherche, publiés ou non, émanant des établissements d'enseignement et de recherche français ou étrangers, des laboratoires publics ou privés. 


\section{A Shock Filter for Image Deblurring and Enhancement with Oriented Hourglass Tensor}

\author{
Magnier Baptiste \\ IMT Mines Alès, LGI2P, \\ 6. av. de Clavières 30100 Alès, France \\ Baptiste.Magnier@mines-ales.fr
}

\author{
Moradi Behrang \\ IMT Mines Alès, LGI2P, \\ 6. av. de Clavières 30100 Alès, France \\ Behrang.Moradi@mines-ales.fr
}

\author{
Walbrecq Cédric \\ IMT Mines Alès, LGI2P, \\ 6. av. de Clavières 30100 Alès, France \\ Cedric.Walbrecq@mines-ales.fr
}

\begin{abstract}
This paper presents a new shock filter for image restoration and enhancement by incorporating diffusion information coming for an hourglass filter. Shock filter represents an important family in the field of non-linear Partial Differential Equations (PDEs) models for image restoration and enhancement. Commonly, the smoothed second order derivative of the image assists this type of method in the deblurring mechanism via of non-linear PDEs models. The advantage of the proposed approach is to insert information issued of a non-linear tensor, i.e., the hourglass filter, in a shock filter process. Technically, the non-linear spatial averaging is more precise to enhance images and sharpen contours efficiently simultaneously. The method is compared to 5 other PDE techniques, presenting its interest, robustness and reliability, as, for example, without creating a grainy effect around deblurred edges by visualizing isophote lines.
\end{abstract}

Index Terms-PDE, shock filter, Hourglass tensor

\section{IntRoduction: IMAGE DEbluRRING AND PDE}

Regularly, digital images are corrupted by blurring due to a movement, focalization problem, unclean optic, image compression or pixel interpolations (up-sampling, rotations...). The common and oldest technique suitable in image deblurring remains the combination of the difference between an original image $I_{0}$ and $\Delta I$ : a blurred version of this same image. Usually, $\Delta I$ corresponds to a blur process equivalent to the heat equation or a convolution of $I_{0}$ with an isotropic Gaussian. This original theory proposed by Gabor is proportional to using the Laplacian operator [9]. Thus, the simplest manner to remove blur in an image is represented by the equation:

$$
\frac{\partial I}{\partial t}=I_{0}-\varepsilon \cdot \Delta I,
$$

where $t$ represents the time or the observation scale and $\varepsilon<1$ is a little scalar to control the deblurring effect. This process is equivalent to the inverse heat equation. However, this technique is not stable because the procedure blows up after several iterations and generates an unusable image [9]. To improve eq. 1, rather than applying a global operator on the whole the image, the main idea is to iterate local operators at level of each pixel. Non-linear Partial Differential Equations (PDEs) may achieve this task [9] [2], practicing anisotropic diffusions of pixel information. Indeed, PDEs belong to one of the most important part of mathematical analysis and are closely related to the physical world. Rather, non-linear PDEsbased diffusion process overcome the undesirable effects of linear smoothing filtering such as smoothing meaningful edges of the image. In this context, images are considered as evolving functions of time and a regularized image $I_{t}$ can be seen as a version of $I_{0}$ at a special scale. Besides, the shock filter theory is based on PDE framework.

The aim of this paper is to incorporate an hourglass filter for an efficient image deblurring process via PDE.

\section{SHOCK Filters: Related WORKS}

This section presents the main PDEs for image deblurring. In order to regularize images by controlling the diffusion, the pioneer work of Perona and Malik on anisotropic diffusion has been one of the most influential papers in the area [14]. Thus, the proposed model is described by the following equation:

$$
\frac{\partial I}{\partial t}(x, y, t)=\operatorname{div}(g(|\nabla I|) \cdot|\nabla I|),
$$

where $|\nabla I|$ represents the modulus of the gradient with mask of type [ -11$]$ and $g$ a decreasing function satisfying $g(0)=1$ and $g(+\infty)=0$; there exists several $g$ functions [2], this function may be:

$$
g(|\nabla I|)=e^{-\left(\frac{|\nabla I|}{K}\right)^{2}},
$$

with $K \in \mathbb{R}_{*}^{+}$a constant that can be assimilated to a gradient threshold or a diffusion barrier, slowing down the diffusion process near edges, i.e., where $|\nabla I|$ is large. Moreover, by developing eq. 2 , it is well known that the diffusion moves backward when $|\nabla I|>K$, creating time-reverse equation (a deblurring effect) called a shock filter allowing edge enhancement, an example is available in Fig. 5(m).

\section{A. Original Shock Filter}

In the PDE framework, the seminal contribution developed by Osher and Rudin [13] is equivalent to eq. 1 , hence, the $2 \mathrm{D}$ formulation is:

$$
\frac{\partial I}{\partial t}=-\operatorname{sign}(\Delta I) \cdot|\nabla I| .
$$

The sign function enable to deblur edges, tuned by the gradient magnitude $|\nabla I|$. The Fig. 1(b)-(c) illustrates this deblurring effect and the edge enhancement. Nevertheless, this PDE produces a dilation/erosion for each pixel, creating a high sensitivity to noise. A number of improvements have been proposed, in particular by convolving $I_{0}$ with a Gaussian. 


\section{B. Shock Filter involving Gaussian}

In order to be more robust to noise, the Gaussian function $G_{\sigma}$ may be convolved with $I_{\eta \eta}$, the second directional derivative of the image in the gradient direction, where $\sigma$ represents the standard deviation of the Gaussian. Coupling diffusion along $\xi$, the edge direction $\left(I_{\xi \xi}\right.$ term [9], [2]) and shock filter, the scheme of Alvarez and Mazorra (AM) is [3]:

$$
\frac{\partial I}{\partial t}=C_{\xi} \cdot I_{\xi \xi}-\operatorname{sign}\left(G_{\sigma} * I_{\eta \eta}\right) \cdot|\nabla I|,
$$

with $G_{\sigma}(x, y)=\frac{1}{2 \pi \sigma^{2}} \cdot \mathbf{e}^{-\frac{x^{2}+y^{2}}{2 \sigma^{2}}}$. Also, $|\nabla I|$ represents the modulus of the gradient with a $3 \times 3$ mask (as Sobel masks), $C_{\xi}$ denotes a control function of the diffusivity, as in eq. 3, $I_{\xi \xi}$ denotes the second derivative in the orthogonal direction of $\eta$, i.e., the edge direction. It corresponds to a pure diffusion in the contour directions, called the curvature equation and is defined by:

$$
I_{\xi \xi}=\frac{I_{x x} \cdot I_{y}^{2}-2 \cdot I_{x} \cdot I_{y} \cdot I_{x y}+I_{y y} \cdot I_{x}^{2}}{I_{x}^{2}+I_{y}^{2}}
$$

Also, $I_{\eta \eta}$ is computed such that:

$$
I_{\eta \eta}=\frac{I_{x x} \cdot I_{x}^{2}+2 \cdot I_{x} \cdot I_{y} \cdot I_{x y}+I_{y y} \cdot I_{y}^{2}}{I_{x}^{2}+I_{y}^{2}} .
$$

Actually, $I_{\xi \xi}$ and $I_{\eta \eta}$ are obtained using the partial derivatives of the image $I$, with respect to $x$ and $y$ :

- $I_{x}=\frac{\partial I}{\partial x}$, the image derivative along the $x$ axis,

- $I_{y}=\frac{\partial I}{\partial y}$, the image derivative along the $y$ axis,

- $I_{x x}=\frac{\partial^{2} I}{\partial x^{2}}$, the 2 nd image derivative along the $x$ axis,

- $I_{y y}=\frac{\partial^{2} I}{\partial y^{2}}$, the 2 nd image derivative along the $y$ axis,

- $I_{x y}=\frac{\partial^{2} I}{\partial x \partial y}$, the crossing derivative of $I$.

Note that the Laplacian can be rewritten in the intrincic coordinates $(\xi, \eta)$ as $\Delta I=I_{\xi \xi}+I_{\eta \eta}$.

Some evolutions of the approach in eq. 5 have been proposed, as in [10], where an isotropic diffusion $(\Delta I)$ is applied concerning small gradients. A PDE technique proposed in [7] weights the diffusion and shock terms in accordance with threshold values of the gradient magnitude. On the other hand, in [4] a combination of non-linear curvature diffusion and shock filter is developed. The gradient modulus of $f(s)=$ $\frac{1}{1+s^{2} / k}$ applied on the gradient magnitude favors or inhibits the shock filter.

The main problem of the based-Gaussian models is the creation of homogeneous blobs in flat noisy regions, as illustrated in Fig. 5(d). Moreover, after a certain number of iterations a corner smoothing could be created, as illustrated in Figs. 1(d) and 2(d). Thus, in Figs. 1(d), edges are enhanced whereas corners of the geometric shapes are over-smoothed.

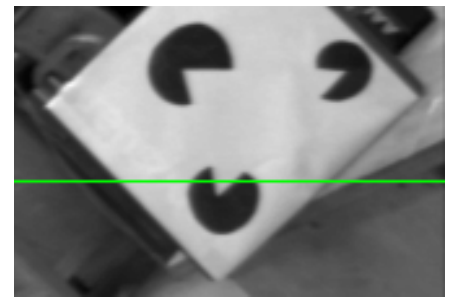

(a) Original image

(c) Signal in (a) vs. signal in (b)

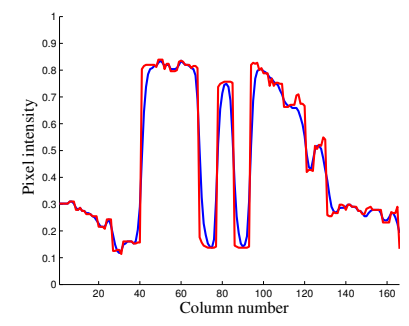

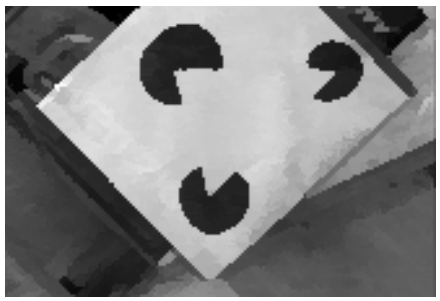

(b) Osher and Rudin [13]

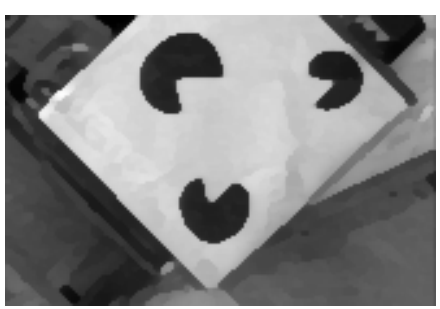

(d) $\mathrm{AM}[3], \sigma=1$
Fig. 1. Illustration of edge improvement using shock filters after 20 iterations. The green line in (a) is tied to the blue curve in (c) and the red curve in (c) represents the same line of pixels in (b) after edge sharpening.

\section{Complex Shock Filter}

A different solution proposed by Gilboa et al. [8] is to change the sign function (cf. eq. 4) in order to take into account both the 2 nd order direction of the second derivative and its magnitude. Denoting $\operatorname{Im}(I)$, the imaginary part of $I$, by using $\frac{2}{\pi} \arctan \left(a \cdot \operatorname{Im}\left(\frac{I}{\theta}\right)\right), a \in \mathbb{R}^{+}$represents the parameter controlling the steepness of the slope of the 2nd order derivative near 0 . When $\theta \in \mathbb{R}_{*}^{+}$tends to $0, \operatorname{Im}\left(\frac{I}{\theta}\right)$ may be considered as the smoothed 2nd order derivative of $I$. The complex shock filter is described by the following equation:

$$
\frac{\partial I}{\partial t}=-\frac{2}{\pi} \arctan \left(a \cdot \operatorname{Im}\left(\frac{I}{\theta}\right)\right) \cdot|\nabla I|+\Lambda \cdot I_{\eta \eta}+\tilde{\Lambda} \cdot I_{\xi \xi},
$$

where $\Lambda$ is a complex diffusion term regularizing the noise and indicating inflection points. $\tilde{\Lambda}$ is a real scalar parameter which corresponds to the amount of diffusion in level-set direction. The real constant $a$ controls the sharpness of the slope of the edge detector near 0 . Using eq. 8, the regions close to contours where the 2 nd order derivative has a higher magnitude, i.e., inflection points will not have equal weights. This translates into a higher deblurring speed near edges and contours than in the flat regions of the image. Despite the originality of complex shock filter and its evolutions, the main drawback of these techniques concerns noisy images. Namely, the deblurring process creates images looking grainy at level of the edges (cf. Fig. 2(e)). In [15], the complex shock method has been improved to limit and correct distortions of edges by incorporating structure tensor and then to diffuse the pixel information along edges (the structure tensor -or gradient tensor- is detailed in next section).

\section{Structure Tensor based Approaches Shock Filter}

The Coherence Enhancing Shock Filter (CESF) has been developed by Weickert [19] with the shock filter theory via 
structure tensor based approach. Thus, the corresponding PDE:

$$
\frac{\partial I}{\partial t}=-\operatorname{sign}\left(\left(G_{\sigma} * I\right)_{\omega_{+} \omega_{+}}\right) \cdot|\nabla I|
$$

allows shock filtering along the direction of the eigenvector $\omega_{+}$ corresponding to the largest eigenvalue of the structure tensor: $J_{\rho}(\nabla I)$. The parameter $\rho$ represents the standard deviation of the Gaussian of the tensor (details in next section, eq. 11).

Tschumperlé and Deriche proposed a different form of the diffusion shock filter, coupling especially for enhancement of color images [17]. This model can be generalized to gray level images using the structure tensor:

$$
\begin{aligned}
\frac{\partial I}{\partial t}= & \varepsilon \cdot\left(I_{0}-I\right)+c_{\omega_{-}} \cdot I_{\omega_{-} \omega_{-}}+c_{\omega_{+}} \cdot I_{\omega_{+} \omega_{+}} \\
& -(1-g(\mathcal{N})) \cdot \operatorname{sign}\left(\left(G_{\sigma} * I\right)_{\omega_{+} \omega_{+}}\right) \cdot|\nabla I|
\end{aligned}
$$

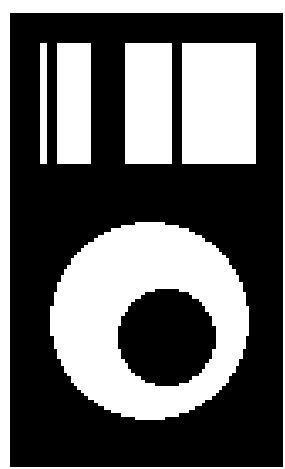

(a) Original image

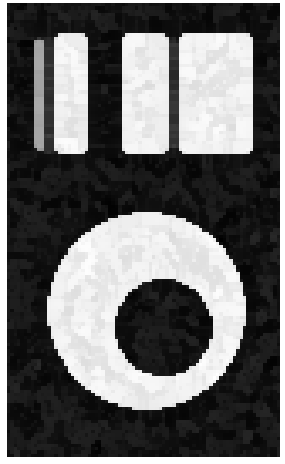

(d) AM [3]

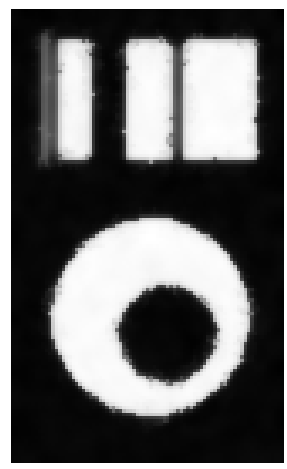

(g) Tschumperlé [17]

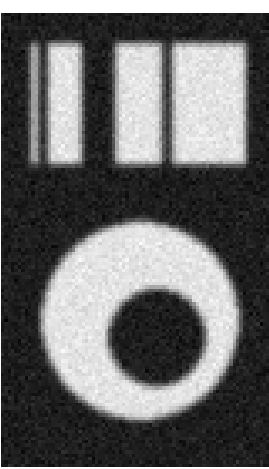

(b) Noisy image

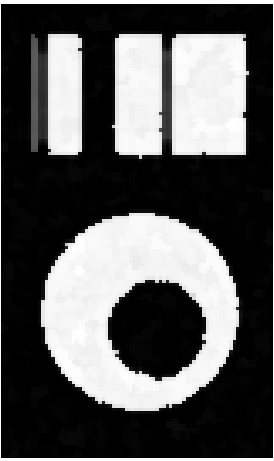

(e) Complex [8]

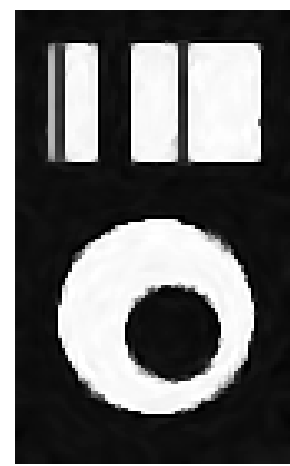

(h) Hourglass tensor

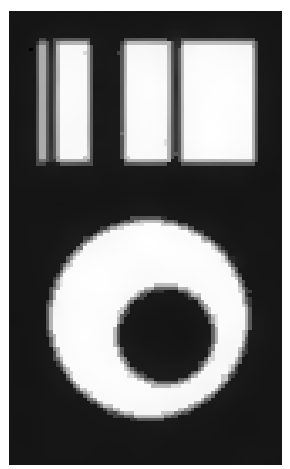

(c) PM [14]

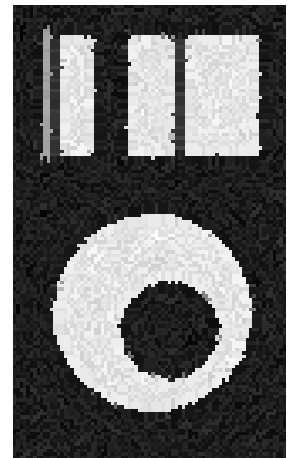

(f) $C E S F[19]$

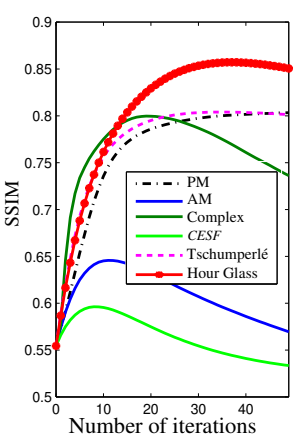

(i) SSIM evolution
Fig. 2. Enhancement of a synthetic blurred and noisy image with $\rho=2$. where $\varepsilon \in \mathbb{R}_{*}^{+}, \quad c_{\omega_{+/-}}$represent two decreasing functions: $c_{\omega_{-}}(\mathcal{N})=\frac{1}{\sqrt{1+\mathcal{N}^{2}}}$ and $c_{\omega_{+}}(\mathcal{N})=\frac{1}{1+\mathcal{N}^{2}}$, with $\mathcal{N}=\sqrt{\lambda_{+}+\lambda_{-}}$ a gradient norm. The shock is controlled by $g(\mathcal{N})$ (cf. eq. 3 ), where $\lambda_{+}$and $\lambda_{-}$denote the two eigenvalues tied to the eigenvectors $\omega_{+}$and $\omega_{-}$respectively, related to the tensor $J_{\rho}$.

These tensorial techniques behave like a contrast enhancing shock filter, they enhance well strip structures, however they may create artificial lines (cf. Fig. 2(f)) or may engender an undesirable grainy effect around edges when dealing with highly noisy images, see Fig. 2(g). On another hand, the main idea in [12] is to insert information issued of oriented half Gaussian kernels in a shock filter process. Notwithstanding a long-term and large scientific effort for PDE-based methods, a satisfactory solution has not been found yet.

\section{The ORIEnTED Hourglass Tensor}

\section{A. Gradient Tensor}

Coming from the first image derivatives, this selection is given by involving a $2 \times 2$ symmetrical structure tensor. The derivation of a scalar image $I$ is called the image gradient and is noted by $\nabla I=\left(I_{x}, I_{y}\right)^{t}$ in which $I_{x}$ and $I_{y}$ represent the image derivatives in $x$ and $y$ directions respectively (usually calculated by means of Gaussian derivative filters with a standard deviation of $\sigma \in \mathbb{R}_{+}$). Particularly, involving a smoothing Gaussian kernel $G_{\rho}$ of standard deviation $\rho$, the first-order gradient tensor -or structure tensor- $J_{\rho}$ is given by:

$$
J_{\rho}(\nabla I)=G_{\rho} * \nabla I \cdot \nabla I^{t}=\frac{1}{2 \pi \rho^{2}} \cdot \mathbf{e}^{-\frac{x^{2}+y^{2}}{2 \rho^{2}}} \cdot\left(\begin{array}{cc}
I_{x}^{2} & I_{x} I_{y} \\
I_{x} I_{y} & I_{y}^{2}
\end{array}\right) .
$$

The scale of the neighborhood information is given by $G_{\rho}$ and the eigenvector $\omega_{+}$of $J_{\rho}$ associated with the largest eigenvalue $\lambda_{+}$is tied to the gradient orientation, as illustrated in Fig. 3(b). When $J_{\rho}$ possesses two positive eigenvalues, then the pixel is considered having at least two distinguished orientations, therefore a corner or a junction [5]; the smallest eigenvalue $\lambda_{-}$may indicate the cornerness measure, as in Fig. 3(c). Consequently, these eigenvalues and eigenvectors are utilized during a PDE process like eq. 9 and 10. However, due to the linear integration caused by the average of $J_{\rho}$, the use of this linear filter leads to an undesirable blurring of structure information. As an example, two close or parallel edges will be merged into a single response, losing the narrow region between them, see Fig. 3(c) for the trace operator $\operatorname{Tr}\left(J_{\rho}(\nabla I)\right)=G_{\rho} * I_{x}^{2}+G_{\rho} * I_{y}^{2}$. Hence, the proposed solution in [11] is to distribute the structure information along the edge using oriented hourglass kernels rather than ellipses [17].

\section{B. Hourglass Tensor}

Considering the edge and gradient directions denoted respectively $\xi$ and $\eta$, the structure information is dispensed along $\xi$ in order to compute a precise diffusion flow. Consequently, the hourglass filter is built such that the angular part which is perpendicular to the edge orientation $\xi$ becomes zero. Thus, 


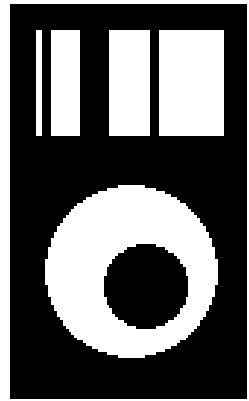

(a) Original image

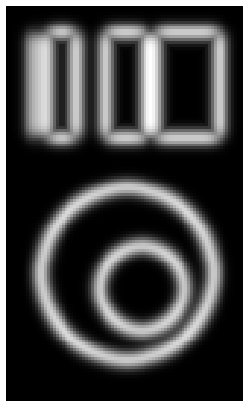

(b) $\lambda_{+}$

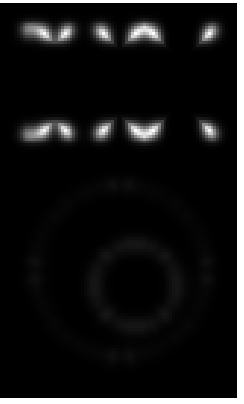

(c) $\lambda_{-}$

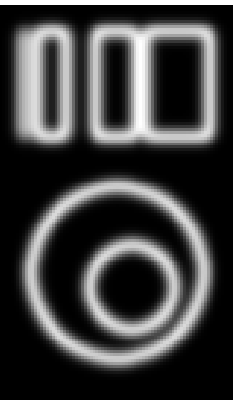

(d) Trace of $J_{\rho}$

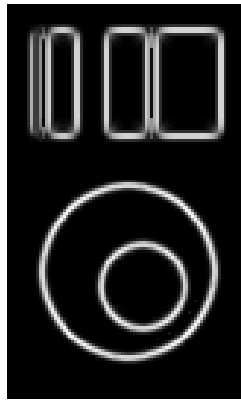

(e) $\mu_{+}$

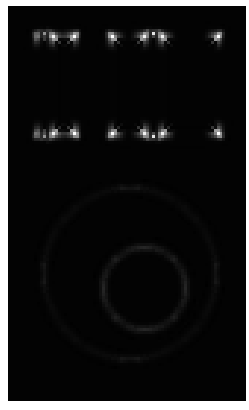

(f) $\mu_{-}$

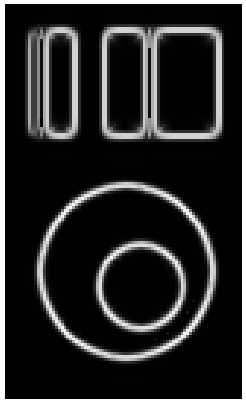

(g) Trace of $\mathcal{H}_{\rho, \gamma, \xi}$

Fig. 3. Eigenvalues and Trace concerning isotropic and Hourglass structure tensors respectively, with $\sigma=1$ and $\rho=2$. For (e)-(g), $\gamma=0.2$.

considering $n=(\cos (\xi), \sin (\xi))^{t}$ and $X$ the pixel coordinates $(x, y)$ the formula is given by:

$$
h_{\rho, \gamma, \xi}(X)=\left\{\begin{array}{l}
\mathbf{e}^{-\frac{X \cdot X^{t}}{2 \rho^{2}}-\frac{1}{2 \gamma^{2}}\left(\frac{n_{\perp} \cdot X^{t}}{n \cdot X^{t}}\right)^{2}}, \quad \text { if } n^{t} \cdot X \neq 0 \\
0, \quad \text { elsewhere. }
\end{array}\right.
$$

The $\gamma$ parameter controls the opening of the angular part, see Fig. 8(a)-(c). Afterward, the filter is normalized such that the sum of all its coefficients is equal to one. Concretely, inspired by the steerable filters [6], at each pixel, the hourglass kernel is rotated in the edge direction $\xi$, according to $n$, as represented in Fig. 4. Then, by convolution the hourglass filter $h_{\rho, \gamma, \xi}$ is applied in a similar way to the structure tensor:

$$
\mathcal{H}_{\rho, \gamma, \xi}(\nabla I)=h_{\rho, \gamma, \xi} * \nabla I \cdot \nabla I^{t}=\left(\begin{array}{ll}
\mathcal{H}_{11} & \mathcal{H}_{12} \\
\mathcal{H}_{21} & \mathcal{H}_{22}
\end{array}\right) .
$$

Thereafter, as for the structure tensor, the highest and lowest eigenvalues respectively denoted $\mu_{+}$and $\mu_{-}$are computed:

$$
\left\{\begin{array}{l}
\mu_{+}=\frac{1}{2}\left(\mathcal{H}_{11}+\mathcal{H}_{22}+\sqrt{\left(\mathcal{H}_{11}-\mathcal{H}_{22}\right)^{2}+4 \cdot \mathcal{H}_{12}^{2}}\right) \\
\mu_{-}=\frac{1}{2}\left(\mathcal{H}_{11}+\mathcal{H}_{22}-\sqrt{\left(\mathcal{H}_{11}-\mathcal{H}_{22}\right)^{2}+4 \cdot \mathcal{H}_{12}^{2}}\right) .
\end{array}\right.
$$

The eigenvalues $\mu_{+/-}$are tied to the gradient and corners. Figs. 3(e)-(g) illustrate the improvement for the computation of the eigenvalues and the trace of the tensor using the hourglass filter. Indeed, close edges are now detected and corners better localized. Information coming from the hourglass tensor can be incorporated in a PDE scheme, where eigenvalues tune the diffusion process in function of the tied eigenvectors. The main purpose of the new regularization PDE flow is to enhance an image without creating a grainy effect around edges.

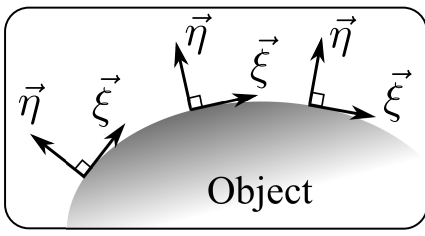

(a) Edge and gradient orientations

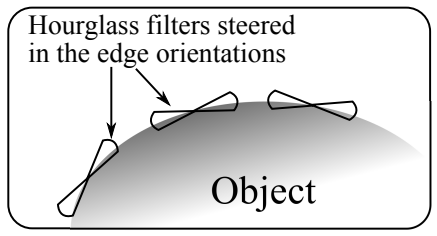

(b) Hourglass kernels
Fig. 4. Hourglass kernels are rotated according to the local edge orientations.

\section{A Shock Filter Process Tuned by the Oriented HOURGLASS TENSOR}

In Fig. 2, several results involving classical methods and structure tensors are presented. Figs. 2(f) and (g) illustrate that thin objects (the vertical line on the top left) is prolonged or merged with the white object on the right. The motivation to use the hourglass filter is to be able to enhance this type of narrow object. Thus, denoting $\mathcal{W}_{+}=\left(\alpha_{+}, \beta_{+}\right)^{t}$ and $\mathcal{W}_{-}=\left(\alpha_{-}, \beta_{-}\right)^{t}$, the two eigenvectors tied to $\mu_{+}$and $\mu_{-}$respectively, the proposed scheme smooths the image in the two orthogonal directions $\mathcal{W}_{+}$and $\mathcal{W}_{-}$, i.e., usually corresponding to the gradient and contour directions respectively. Consequently, $I_{\mathcal{W}_{-}} \mathcal{W}_{-}$ensures an edge preservation whereas $I_{\mathcal{W}_{+}} \mathcal{W}_{+}$helps to create homogeneous regions. They are computed with the second derivatives of the image $I_{x x}$ (in $x$ direction), $I_{y y}$ (in $y$ direction) and $I_{x y}$ (crossing derivative):

$$
\left\{\begin{array}{l}
I_{\mathcal{W}_{+} \mathcal{W}_{+}}=\alpha_{+}^{2} \cdot I_{x x}+2 \cdot \alpha_{+} \cdot \beta_{+} \cdot I_{x y}+\beta_{+}^{2} \cdot I_{y y} \\
I_{\mathcal{W}_{-} \mathcal{W}_{-}}=\alpha_{-}^{2} \cdot I_{x x}-2 \cdot \alpha_{-} \cdot \beta_{-} \cdot I_{x y}+\beta_{-}^{2} \cdot I_{y y}
\end{array}\right.
$$

Now the use of the "sign" function enables a couple equation taking into account the regularization and shock process for image denoising and edge sharpening. The weighting function in eq. 3 is added in order to adapt the intensity of the shock filter, allowing to enhance edges while preserving homogeneous regions. Thus, the proposed regularization PDE flow is given by:

$$
\begin{aligned}
\frac{\partial I}{\partial t} & =C_{\mathcal{W}_{+}} I_{\mathcal{W}_{+}} \mathcal{W}_{+}+C_{\mathcal{W}_{-}} \cdot I_{\mathcal{W}_{-} \mathcal{W}_{-}} \\
& +(g(\mathcal{A})-1) \cdot \operatorname{sign}\left(I_{\mathcal{W}_{+}} \mathcal{W}_{+}\right) \cdot|\nabla I|
\end{aligned}
$$

with:

$$
\begin{aligned}
& \text { - } \mathcal{A}=\mu_{+}+\mu_{-}, \\
& \text {- } C_{\mathcal{W}_{+}}(\mathcal{A})=\frac{1}{1+\mathcal{A}}, \\
& \text { - } C_{\mathcal{W}_{-}}(\mathcal{A})=\frac{1}{\sqrt{1+\mathcal{A}}} .
\end{aligned}
$$

The gradient norm $\mathcal{A}$ is chosen because it detects as well edges as corners, as shown in Fig. 3(e)-(f). Further, to ensure performance of the coupled diffusion and shock process, $C_{\mathcal{W}_{+}}$ and $C_{\mathcal{W}_{-}}$, with $C_{\mathcal{W}_{+}}(\mathcal{A})<C_{\mathcal{W}_{-}}(\mathcal{A})$ are two decreasing functions. Indeed, if the diffusion along $\mathcal{W}_{+}$is too important, edges will be blurred, which is contrary to the desirable effect. 


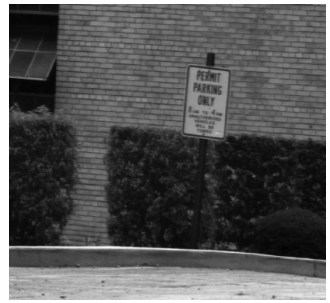

(a) Original image

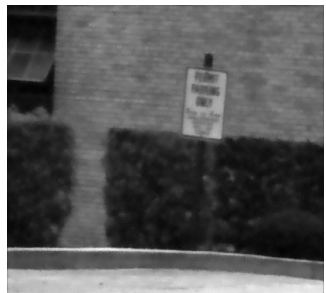

(f) Bilateral filter [16]

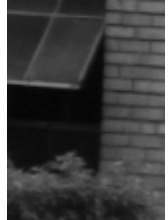

(k) enlarg. of (a)

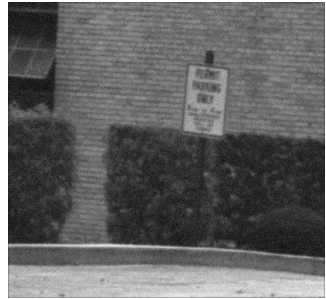

(b) Noisy and blurred image

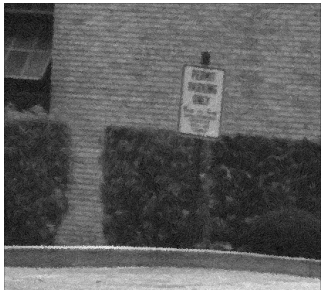

(g) CESF [19]

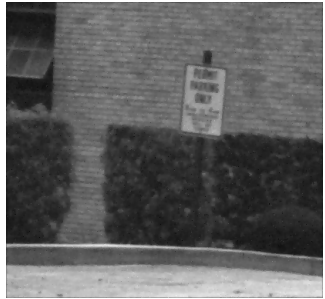

(c) PM [14]

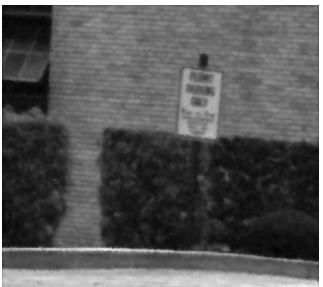

(h) Tschumperlé [17]

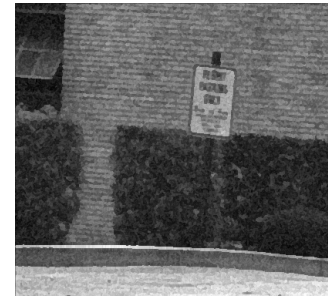

(d) AM [3]

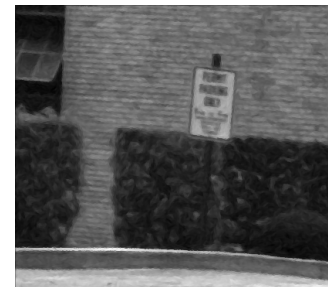

(i) Hourglass tensor

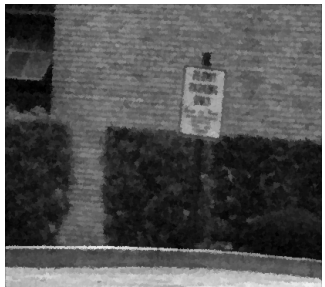

(e) Complex [8]

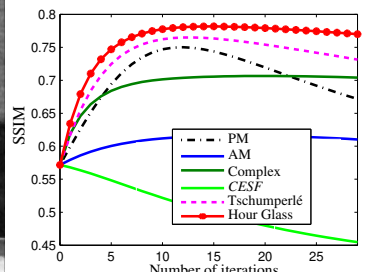

(j) SSIM evolution

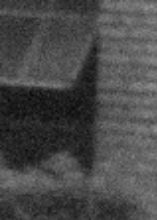

(1) enlarg. of (b)

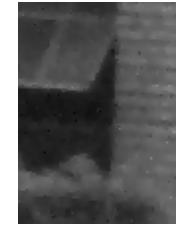

(m) enlarg. of (c)

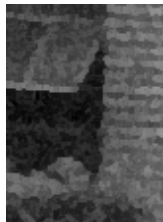

(n) enlarg. of (d)

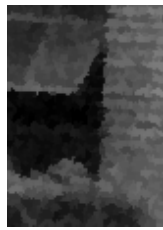

(o) enlarg. of (e)

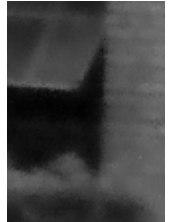

(p) enlarg. of (f)

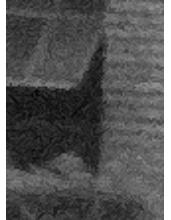

(q) enlarg. of (g)

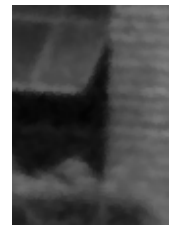

(r) enlarg. of (h)

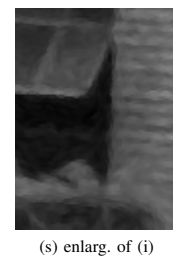

\section{Evaluation And Experimental Results}

Several results of the proposed PDE are presented in this section. These results are compared to the different approaches described above. For the evaluation in Figs. 2 and 5, as far as the evaluation process is concerned, the Structural Similarity Index Measure (SSIM) [18] is reported and plotted as a function of the number of iterations for each method. To shed light on the effectiveness of the proposed shock filter, two tested images are noised by white Gaussian noise $(\sigma=10)$ and blurred using a convolution with a Gaussian $(\sigma=1)$, cf. Figs. 2 and 5. Then they are independently treated by PeronaMalik (PM) [14] , Alvarez-Mazorra (AM) [3] , Complex [8], Weickert [19] and Tschumperlé et al. [17] methods. The parameter for methods involving Gaussians is $\sigma=1$; for complex shock filters, $\theta=0.001, a=2$ and $\hat{\lambda}=\lambda=0.1$. Finally, the presented experimental results and images resulting the different methods are tied to the best SSIM score.

The first result is presented in Fig. 2. As stated above, the considered image is composed of geometrical features and close objects that need to be deblurred and enhanced. The new approach outperforms classical shock filter methods in term of SSIM values (with default parameters). Visually, objects are separated (even narrow regions) and the majority of edges are straight, with desired homogeneous regions, contrary to other results. For the second experiment concerning a real image in Fig. 5, SSIM scores of the proposed technique remain above other scores. Results are also compared with the wellknown bilateral filter (sharp edge-preserving filter), where the standard deviation of the spatial Gaussian smoothing for this filter is the same than the tensor parameter $(\sigma=\rho=3)$. As the enlargements (enlarg.) show in Fig. 5(k)-(s), AM and complex methods create homogeneous blobs in flat noisy regions, CESF is disturbed by the level of noise, creates stripes, whereas PM, bilateral and Tschumperlé et al. approaches blur the textures. On the contrary, the proposed technique is able to improve the textures of the bricks and of the bushes as the majority of the main structures. Moreover, the new regularization PDE flow along the eigenvectors enables an image enhancement without creating a grainy effect around edges.

Fig. 6 illustrates enhancements of a real image using two different tensors (linear and non-linear). In this way, the regularizing flow using linear spatial averaging $\left(J_{\rho}\right.$ in eq. 11)

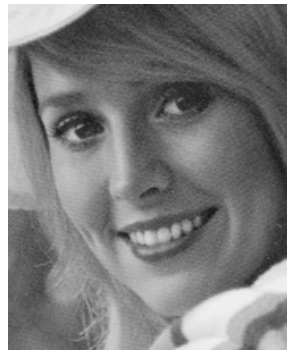

(a) Image $176 \times 221$

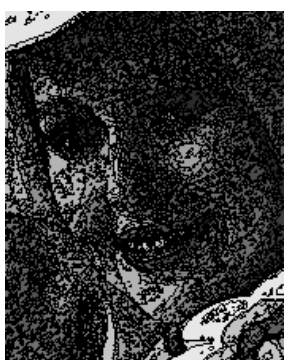

(d) Isophote of (a)

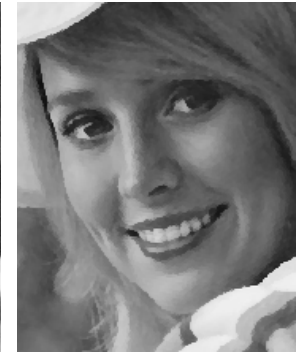

(b) Isotropic tensor [17]

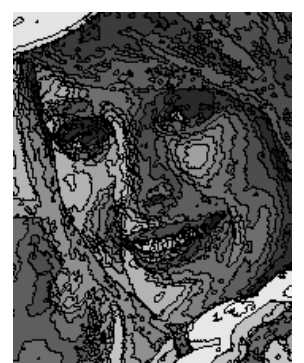

(e) Isophote of (b)

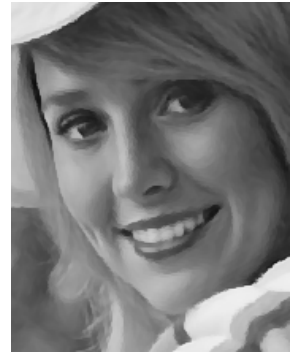

(c) Hourglas tensor

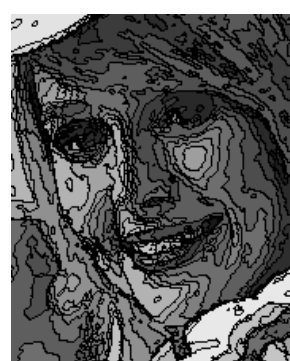

(f) Isophote of (c)
Fig. 6. Enhancement of a real image (Elaine) and comparison of isophote lines on 16 levels. Results obtained after 30 iterations with $\rho=3$ for both. 


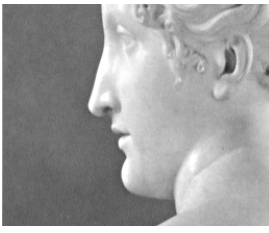

(a) Image $176 \times 221$

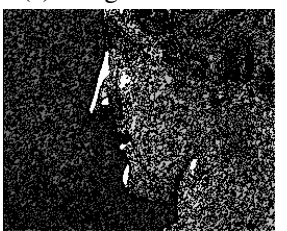

(d) Isophote of (a)

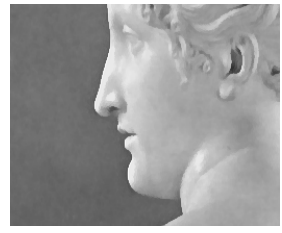

(b) Isotropic tensor [17]

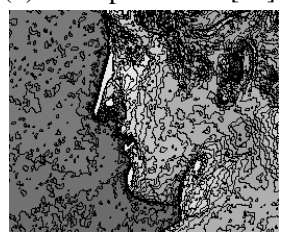

(e) Isophote of (b)

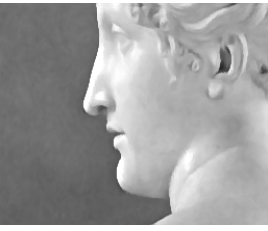

(c) Hourglas tensor

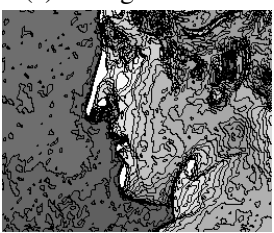

(f) Isophote of (c)
Fig. 7. Enhancement of a real image (Paulina) and comparison of isophote lines on 32 levels. Results obtained after 20 iterations with $\rho=3$ for both.

and non-linear $\left(\mathcal{H}_{\rho, \gamma, \xi}\right.$ in eq. 13)) are compared together by visualizing isophote (lines of constant pixel intensities on 16 levels). Choosing the same parameters for both models, this showcases the grainy effect around edges which are visible in Fig. 6(e) for the linear method. On the contrary, contours are enhanced by using the hourglass filter; they are straighter, as for the texture of hairs (Fig. 6(f)). Inside flat regions, isophote lines are less winding than the image restoration using linear tensor, showing the efficiency of the proposed regularization PDE flow. In the same manner, plotted isophotes on 32 levels is Fig. 7 are more regular with the hourglass filter-based shock filter model. Eventually, results presented in Fig. 9 showcase small objects and the stripes created by a brush for a painting.

\section{CONCLUSION AND Discussion}

A new hourglass filter-based shock filter model has been introduced in this paper. The hourglass tensor allows to integrate narrow objects having close edges and then detect their contours in order to improve the deblurring effect via a PDE approach. Contrary to existing shock filters, it avoids a grainy effect at level of deblurred contours whereas flat regions are regularized without create undesirable blobs areas.

Concerning the $\gamma$ parameter in eq. 12 controlling the opening of the angular part (see Fig. 8(a)-(c) with $\rho=4$ ), when $\gamma$ is too large, the diffusion flow along $\mathcal{W}_{-}$using $\sigma=1$ leads to a leakage of pixel information, especially at the level of corners and close objects. As stated in [11], the $\gamma$ parameter should be as small as possible to obtain pronounced orientedness. Regarding the proposed regularization PDE flow, the remark is the same for the enhancement of narrow and close objects.

Future works may concern the extension of the hourglass tensor to handle $3 \mathrm{D}$ images or in the $4 \mathrm{D}$ ray space [1].

\section{REFERENCES}

[1] P. Allain, L. Guillo and C. Guillemot, "Light field denoising using 4D anisotropic diffusion,” IEEE ICASSP, pp. 1692-1696, 2019.

[2] G. Aubert and P. and Kornprobst, Mathematical problems in image processing: partial differential equations and the calculus of variations (second edition). vol. 147, Springer-Verlag, 2006.

[3] L. Alvarez and L. and Mazorra, "Signal and image restoration using shock filters and anisotropic diffusion," SIAM J. Numer. Anal. 31(2), pp. 590-605, 1994.

[4] S. Bettahar, P. Lambert and A.B. Stambouli, "Anisotropic color image denoising and sharpening," IEEE ICIP, pp. 2669-2673, 2014.

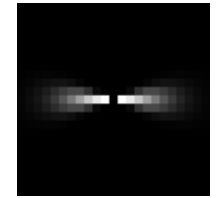

(a) $h_{\rho, \gamma, \xi}$, with $\gamma=0.2$

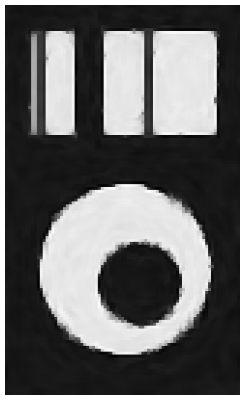

(d) 20 iterations, $\gamma=0.2$
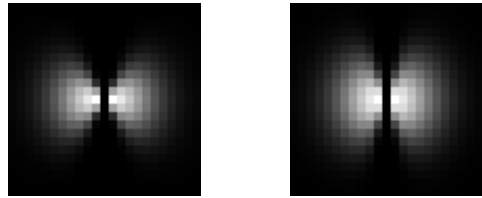

(b) $h_{\rho, \gamma, \xi}$, with $\gamma=1.1$ (c) $h_{\rho, \gamma, \xi}$, with $\gamma=2.1$
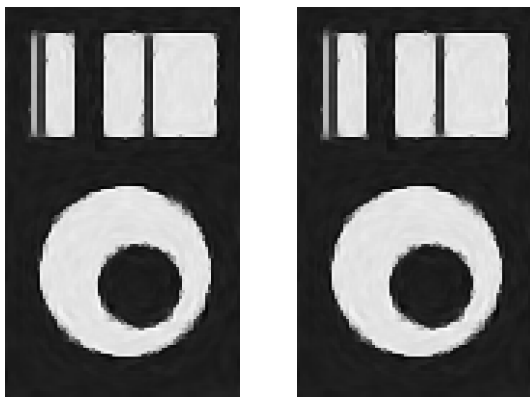

(e) 20 iterations, $\gamma=1.1$ (f) 20 iterations, $\gamma=2.1$
Fig. 8. Comparison of the regularization PDE flow presented in eq. 16 using different values of $\gamma$, with $\rho=4$. The noisy image is available in Fig. 2(b).

[5] W. Förstner, "A feature based correspondence algorithm for image matching," Int. Archives of Photogrammetry and Remote Sensing, 26(3), pp. 150-166, 1986.

[6] W. T. Freeman and E. H. Adelson, "The design and use of steerable filters," IEEE TPAMI, 13(9), pp. 891-906, 1991.

[7] S. Fu, Q. Ruan, W. Wang and J. Chen, "Region-based shock-diffusion equation for adaptive image enhancement," Advances in Machine Vision, Image Processing, and Pattern Analysis, Springer, pp. 387-395, 2006.

[8] G. Gilboa, A. Sochen and Y. Zeevi, "Regularized Shock Filters and Complex Diffusion,” IEEE ECCV, Part I, pp. 399-413, 2002.

[9] F. Guichard, L. Moisan and J.M. Morel, "A review of PDE models in image processing and image analysis," J. de Physique IV, 12(1), pp. 137-154, 2002.

[10] P. Kornprobst, R. Deriche and G. Aubert, "Image coupling, restoration and enhancement via PDE's," IEEE ICIP, pp. 458-46, 1997.

[11] U. Köthe, "Edge and junction detection with an improved structure tensor," Joint Patt. Rec. Symp., pp. 25-32, Springer, 2003.

[12] B. Magnier, V. Noblet, A. Voisin and D. Legouestre, D. "Derivative Half Gaussian Kernels and Shock Filter," ACIVS, pp. 584-597, 2018.

[13] S. Osher and L.I. Rudin, "Feature-oriented image enhancement using shock filters," SIAM J. Numer. Anal. 27(4), pp. 919-940, 1990.

[14] P. Perona and J. Malik, J., "Scale-space and edge detection using anisotropic diffusion,” IEEE TPAMI, 12, pp. 629-639, 1990.

[15] R. Terebes, M. Borda, C. Germain and O. Lavialle, "A novel shock filter for image restoration and enhancement," EUSIPCO, pp. 255-259, 2012.

[16] C. Tomasi and R. Manduchi, "Bilateral filtering for gray and color images," IEEE ICCV, pp. 839-846, 1998.

[17] D. Tschumperlé and R. Deriche, "Diffusion PDE's on vector-valued images: local approach and geometric viewpoint," IEEE Signal Processing Magazine, 19(5), pp. 16-25, 2002.

[18] Z. Wang, A. Bovik, H. Sheikh and E. Simoncelli, "Image quality assessment: from error visibility to structural similarity," IEEE TIP, 13(4), pp. 600-612, 2004.

[19] J. Weickert, “Coherence-enhancing shock filters," LNCS, pp. 1-8, 2003.

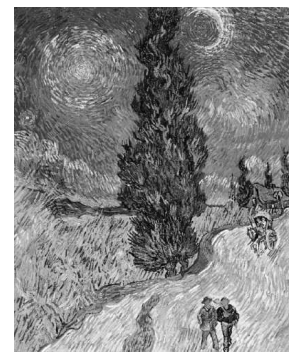

(a) Picture of van Gogh paint

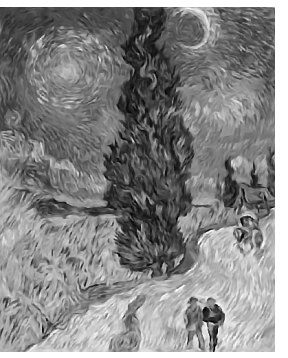

(b) 50 iterations

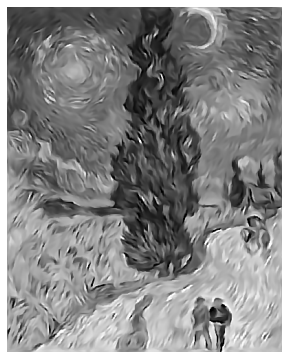

(c) 150 iterations
Fig. 9. Exemple of the regularizing flow, $\sigma=0.7, \rho=3$ and $\gamma=0.2$. 